\title{
Perspective Piece \\ Resilient Clinical Trial Infrastructure in Response to the COVID-19 Pandemic: Lessons Learned from the TOGETHER Randomized Platform Clinical Trial
}

\author{
Jamie I. Forrest, ${ }^{1,2}$ Angeli Rawat, ${ }^{2}$ Felipe Duailibe, ${ }^{2}$ Christina M. Guo, ${ }^{1,3}$ Sheila Sprague, ${ }^{4}$ Paula McKay, ${ }^{5}$ \\ Gilmar Reis, ${ }^{5,6}$ and Edward J. Mills ${ }^{1,4 *}$ \\ ${ }^{1}$ Platform Life Sciences, Vancouver, Canada; ${ }^{2}$ University of British Columbia, Vancouver, Canada; ${ }^{3}$ University of Aberdeen, Aberdeen, Scotland; \\ ${ }^{4}$ McMaster University, Hamilton, Canada; ${ }^{5}$ CardResearch, Belo Horizonte, Brazil; ${ }^{6}$ Pontifica Católica Universidade de Minas Gerais, Belo \\ Horizonte, Brazil
}

\begin{abstract}
In response to the COVID-19 pandemic, clinical research groups across the world developed trial protocols to evaluate the safety and efficacy of treatments for COVID-19. Despite this initial enthusiasm, only a small portion of these protocols were implemented. Of those implemented, a fraction successfully recruited their target sample size to analyze and disseminate findings. More than a year and a half into the COVID-19 pandemic, only a few clinical trials evaluating treatments for COVID-19 have generated new evidence. Productive randomized platform clinical trials evaluating COVID-19 treatments may attribute their success to intentional investments in developing resilient clinical trial infrastructures. Health system resiliency discourse provides a conceptual framework for characterizing attributes for withstanding shocks. This framework may also be useful for contextualizing the attributes of productive clinical trials evaluating COVID-19 therapies. We characterize the successful attributes and lessons learned in developing the TOGETHER Trial infrastructure using a health system resiliency framework. This framework may be considered by clinical trialists aiming to build resilient trial infrastructures capable of responding rapidly and efficiently to global health threats.
\end{abstract}

In 2020, clinical trial researchers developed protocols to evaluate the safety and effectiveness of potential treatments for COVID-19. Despite more than 3,000 trials registered (as of November 2021), few have generated findings, with the exception of smaller randomized controlled trials. ${ }^{1}$ Platform trials (e.g., SOLIDARITY, ${ }^{2}$ RECOVERY, ${ }^{3-5}$ Platform Randomised trial of INterventions against COVID-19 In older peoPLE [PRINCIPLE], ${ }^{6-8}$ REMAP-CAP, ${ }^{9,10}$ and TOGETHER, ${ }^{11,12}$ ) hold the most promise for the perpetual evaluation of COVID19 therapeutics. The PRINCIPLE and TOGETHER Trials were similarly successful in recruiting patients and generating evidence, as compared and contrasted in Table 1. Although many similarities exist between these two trials, both of which focused on outpatient high-risk populations in community settings, the settings were different. The TOGETHER Trial took place in one state of a middle-income country (Brazil) with a more fragmented health system compared with the PRINCIPLE Trial, which took place within a well-integrated health system with a national health insurance scheme. The successes of these trials may be attributed to investments in a resilient clinical trial infrastructure. A clinical trial infrastructure (defined as the human, material, and knowledge networks that form a responsive implementation of productive trial protocols) must be resilient to threats (e.g., COVID-19), and is essential when barriers to sustainable funding are common (academic and public sectors). ${ }^{13}$

Health system resiliency provides a conceptual framework to evaluate and guide reforms during and after shocks (e.g., pandemics, natural disasters, political turmoil). ${ }^{14}$ The framework considers five domains: 1) aware (apply the bestknown emerging evidence to inform decision making), 2) diverse (provide multiple pathways for patients and providers

*Address correspondence to Edward J. Mills, Department of Health Research Methods, Evidence, and Impact, Faculty of Health Sciences, McMaster University, 1280 Main St. West, Hamilton, Ontario, L8S 4L8, Canada. E-mail: millsej@mcmaster.ca with various skills), 3) self-regulating (identify and re-allocate resources as needed), 4) integrated (draw efficiently on resources and expertise from other sectors as needed), and 5) adaptive (be able to change in response to new information).

This framework may be useful in characterizing productive clinical trial networks and infrastructure in responding to threats such as COVID-19. The TOGETHER Trial is an adaptive platform randomized clinical trial to evaluate re-purposed therapies for the treatment of early-diagnosed COVID-19 in the Minas Gerais state of Brazil. ${ }^{12}$ The ongoing trial (as of November 2021) has enrolled more than 3,800 patients and has published findings on the safety and effectiveness of treatment of early-diagnosed outpatient clinical management of COVID-19 with lopinavir/ritonavir or hydroxychloroquine, ${ }^{11}$ and fluvoxamine,$^{12}$ with analyses of metformin, ivermectin, doxazosin, and peginterferon lambda pending (Figure 1). The TOGETHER Trial consortium is a network of investigators affiliated with academic institutions in Canada, Brazil, and the United States, in partnership with contracted private companies providing data management and analytical support.

We describe the attributes of the TOGETHER Trial within the framework of health system resiliency. We identified four attributes of the TOGETHER Trial related to clinical trial infrastructure resiliency: 1) participants and partners; 2) knowledge, communication and evidence generation; 3) health system capacity and resourcing; and 4) governance, administration, and financing. By documenting these lessons learned, we demonstrate the utility of this framework as it applies to the development of a clinical trial infrastructure responsive to emergent global health threats (Figure 2).

Participants and partners refers to patient engagement and partnerships across the trial. In-person participant recruitment and monitoring were limited by the infectiousness of COVID-19 and the rapidly changing government lockdown scenarios limiting participants' mobility. We used social media (WhatsApp, the most popular messaging app 
TABLE 1 Comparing and contrasting the TOGETHER and PRINCIPLE trials

\begin{tabular}{|c|c|c|}
\hline Characteristic & TOGETHER trial & PRINCIPLE trial \\
\hline Location & Brazilian state of Minas Gerais & United Kingdom \\
\hline Patient population & $\begin{array}{l}\text { Community setting, patients are at least } 18 \\
\text { years of age, have a positive antigen test } \\
\text { for severe acute respiratory syndrome } \\
\text { coronavirus } 2 \text {, and have an indication for } \\
\text { high risk of disease severity, including } \\
\text { comorbidities, older age, or high body } \\
\text { mass index }\end{array}$ & $\begin{array}{l}\text { Community setting, patients are } 50 \text {-year- } \\
\text { olds at high risk of complications (with } \\
\text { comorbidities) or } 65 \text {-year-olds or older } \\
\text { who were unwell for up to } 14 \text { days with } \\
\text { suspected COVID-19 }\end{array}$ \\
\hline Date recruitment began & June 2020 & April 2, 2020 \\
\hline $\begin{array}{l}\text { No. of participants recruited (November } \\
\text { 2021) }\end{array}$ & 3,800 & 7,833 \\
\hline Types of recruitment & $\begin{array}{l}\text { Patients presenting to an outpatient clinic } \\
\text { setting with clinical criteria for } \\
\text { presumptive diagnosis of COVID-19 who } \\
\text { met the eligibility criteria were invited to } \\
\text { participate. }\end{array}$ & $\begin{array}{l}\text { Twenty-five percent of participants were } \\
\text { from } 200 \text { general practices; } 75 \% \text { were } \\
\text { from online self-referral. }\end{array}$ \\
\hline $\begin{array}{l}\text { Strategies to reduce person-to-person } \\
\text { contact }\end{array}$ & $\begin{array}{l}\text { WhatsApp messaging and video for } \\
\text { recruitment, communication with trial } \\
\text { coordinators, and monitoring of } \\
\text { participants at } 13 \text { clinical research sites; } \\
\text { hotline }\end{array}$ & $\begin{array}{l}\text { Online self-referral; participants received } \\
\text { trial therapeutics via courier }\end{array}$ \\
\hline No. of interventions & 7 & 6 \\
\hline $\begin{array}{l}\text { Therapeutics trialed vs. placebo or standard } \\
\text { of care }\end{array}$ & $\begin{array}{l}\text { Doxazosin, fluvoxamine, } \\
\text { hydroxychloroquine, ivermectin, lopinavir/ } \\
\text { ritonavir, metformin, peginterferon } \\
\text { lambda }\end{array}$ & $\begin{array}{l}\text { Azithromycin, budesonide (inhaled), } \\
\text { colchicine, doxycycline, favipiravir, } \\
\text { ivermectin }\end{array}$ \\
\hline $\begin{array}{l}\text { Findings with promise for potential } \\
\text { therapeutic value }\end{array}$ & $\begin{array}{l}\text { Fluvoxamine reduced the chance of COVID- } \\
\text { 19-related hospitalization. }\end{array}$ & $\begin{array}{l}\text { Inhaled budesonide improved time to } \\
\text { recovery, with the potential to reduce } \\
\text { hospital admissions or deaths. }\end{array}$ \\
\hline
\end{tabular}

in Brazil) to recruit patients and connect them with trial coordinators, and to remind clinicians to follow up with patients and monitor patients (including adverse events) via video calls in lieu of in-person monitoring. Participants could also call a hotline to access clinicians and trial staff quickly.

Fluctuations in clinical trial human resources during a pandemic required rapid responses and innovative partnerships.
We engaged experts and diverse stakeholders, including academics, pharmacists, clinicians, and statisticians, and experts in methodology, knowledge translation, guideline development, and ethics management. We expanded traditional partnerships and collaborations to new partners and other clinical trials, which improved our name recognition and access to experts, and diversified our resources.

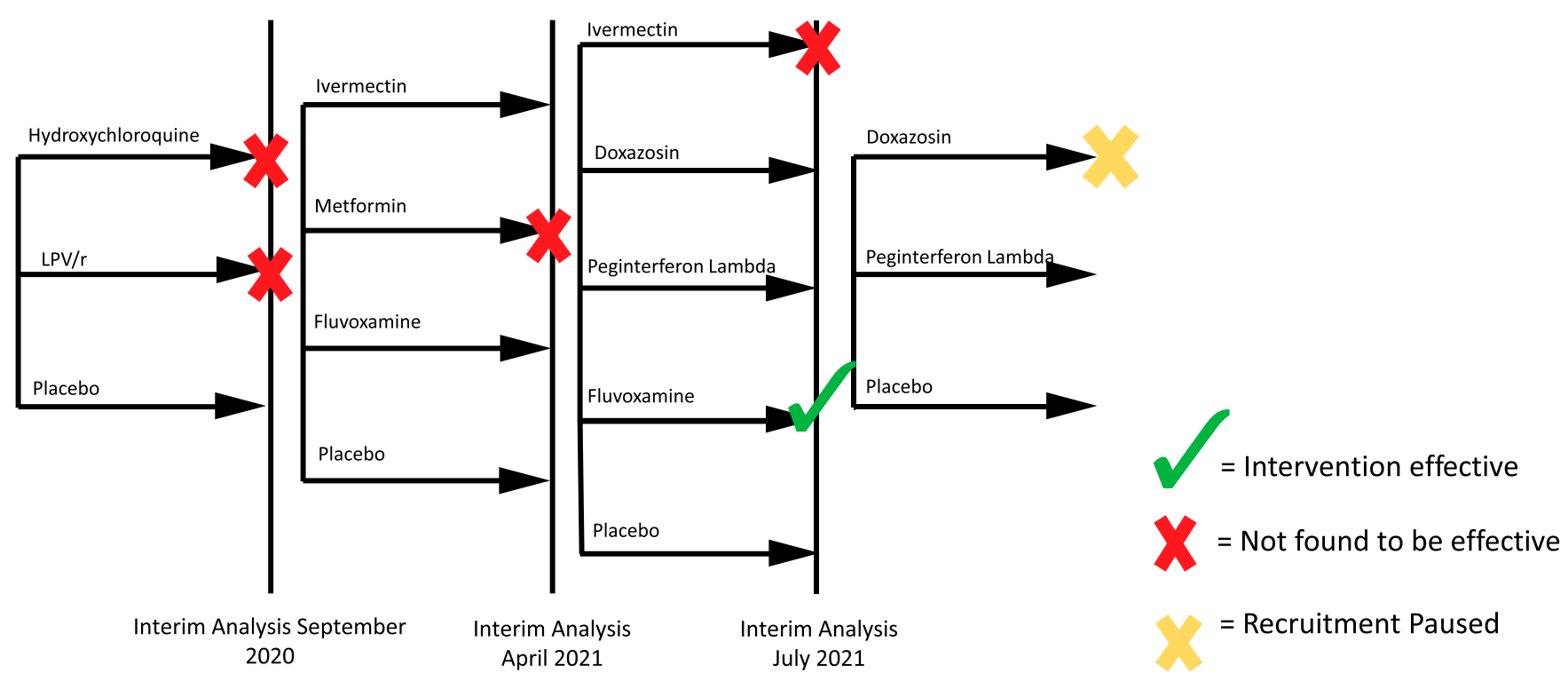




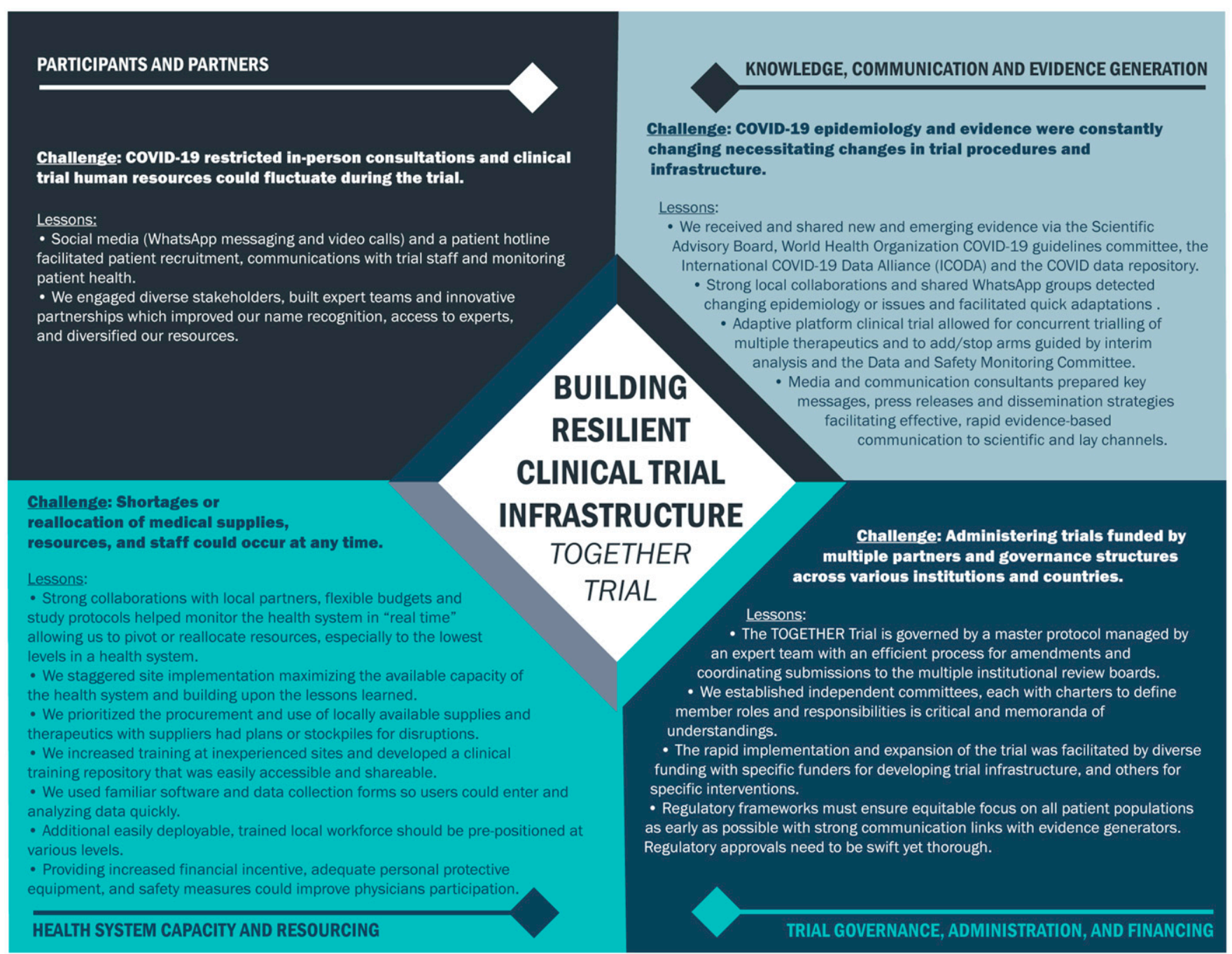

FIGURE 2. Summary of lessons learned.

Knowledge, communication, and evidence generation refers to how knowledge was generated, used, communicated, and disseminated. The ever-changing epidemiology and evidence related to COVID-19 challenged resilient clinical trial infrastructures. We had confidence in the evidence we received via our scientific advisory board, which reviewed new and emerging evidence regularly, and by having links with the WHO COVID-19 guidelines committee. Quick data sharing locally was facilitated by strong publicsector partnerships. We learned quickly from other evidence generators globally and shared our findings via the International COVID-19 Data Alliance and the COVID data repository.

With timeliness being critical in building a resilient trial infrastructure, we detected problems or changes early, shared information, and adapted quickly (e.g., shifting recruitment and clinical capacity as incidence rates changed). Strong collaborations with local partners monitored changing epidemiology in "real time" and facilitated quick responses. Shared WhatsApp groups within the steering committee and for each clinic allowed discussions to solve problems and identify issues early. Similar time zones facilitated knowledge sharing, and a designated interpreter ensured effective communication across languages. In collaborations with vastly different time zones, designated trial liaisons could function as on-call personnel responding to urgent requests outside of business hours.

One advantage of the adaptive platform clinical trial was that we trialed multiple investigational products simultaneously with the capacity to stop trial arms as evidence emerged of low or no efficacy, or to add potential therapeutics. Conducting interim analyses allowed us to detect changes at predetermined time points during the trial. The Data and Safety Monitoring Committee was flexible and advised rapidly on any adaptations (e.g., when to drop or add arms). Misinformation of study results and/or the politicization of therapeutics under investigation could impact trial participation and evidence uptake. For example, some patients refused participation, knowing they may be randomized to treatment with hydroxychloroquine-a politicized treatment of COVID-19 in Brazil. ${ }^{15}$ Having designated media and communication consultants who scanned online spaces constantly ensured our evidence was represented correctly. They also prepared key messages and dissemination strategies facilitating effective, evidence-based communication to scientific and lay channels. Releasing scientific 
evidence via press releases allowed for rapid evidence dissemination to meet the urgency and high demand for knowledge.

A major consideration for building resilience is the fluctuating health system capacity and resources available for clinical trials. Shortages or reallocation of medical supplies, resources, and staff could occur at any time. Strong collaborations with local partners monitored the health system in real time, allowing us to pivot or reallocate resources accordingly. Flexible budgets facilitated quick resource reallocation to all sectors of the health system participating in the trial. To accommodate changing human resource needs, an extra easily deployable, trained local workforce should be pre-positioned at various levels. All staff are provided with ongoing training, support, and supervision so they are current with procedures and protocols, further ensuring versatility in their job functions.

Supply chains could be disrupted and import channels could be limited, potentially leading to demand-driven price increases and limited or unavailable supplies. We first implemented in sites experienced with clinical trials and staggered site implementation thereafter to ensure we met the available capacity of the health system and built upon our lessons learned. We prioritized the procurement and use of locally available supplies and therapeutics or those procured from countries with no transfer or travel restrictions between them and Brazil. We ensured the suppliers had plans or stockpiles for disruptions. We did not anticipate the high costs for complex laboratory testing resulting from a lack of supply globally, and we recommend flexible study protocols and budgets to accommodate shocks in pricing.

Engaging diverse clinical sites also meant having disparities in human resource capacities and clinical trial experience. We increased training at inexperienced sites and developed a clinical training repository that was easily accessible and shareable. Resilient referral networks were built, where participants were monitored frequently by physicians via WhatsApp video calls, and were linked quickly and referred to larger hospitals. By using familiar software and data collection forms, and ensuring users' trainings were upto-date, we avoided steep learning curves that prevent users from entering and analyzing data quickly. Physicians could be deterred from participating in trials when the disease transmission is novel or the populations are in high-risk areas. Providing an increased financial incentive, adequate personal protective equipment, and safety measures could improve participation.

Trial governance, administration, and financing must also be resilient. The primary attribute of a resilient platform trial is the execution of a master protocol, detailing trial procedures for the evaluation of multiple interventions to treat the same disease within a single overall trial structure. ${ }^{16}$ The TOGETHER Trial is governed by a master protocol that is flexible to trial adaptations, including rules for adding and dropping arms. The master protocol for the TOGETHER Trial was managed by an expert team affiliated with the sponsor institution and the institutional review board of record, which fostered an efficient process for amending the master protocol and coordinating submissions to the multiple institutional review boards that govern the trial.

Establishing independent committees, each with charters to define member roles and responsibilities, is critical. The
TOGETHER Trial developed charters for the following committees: scientific steering, central trial coordination, local trial implementing, data and safety monitoring, and event adjudication. For example, the local implementing committee established memoranda of understandings with each clinical site, which facilitated the rapid expansion of the trial network throughout the state of Minas Gerais.

Diversifying funding is important for sustaining resilient clinical trial networks. The TOGETHER Trial is funded by private donors and philanthropic organizations that accommodated the unique limitations and scientific priorities of each party. We secured funding dedicated to developing clinical trial infrastructure, whereas other funders supported the trial activity implementation for specific interventions. Although patchwork funding from diverse sources has facilitated the rapid implementation and expansion of the TOGETHER Trial, a transition to more sustainable and long-term funders is essential to support the perpetual evaluation of potential interventions.

Evidence generated from such trials can only be as effective as the systems in place to uptake the evidence quickly. Early in the COVID-19 response, evidence generated from inpatient trials were prioritized. We missed an opportunity to generate concurrently evidence for outpatient populations. Although outpatients have a lower probability of developing severe illness, interventions could greatly reduce the need for health system resources. ${ }^{17}$ Therefore, we recommend regulatory frameworks ensure an equitable focus on all patient populations as early as possible, with strong communication links with evidence generators, and regulatory and implementing authorities. Regulatory approvals need to be swift yet thorough.

To our knowledge, this is the first documentation of a platform trial infrastructure in the COVID-19 era and the first to characterize attributes of the trial infrastructure within an analogous framework to health system resiliency. We identified four attributes for a resilient clinical trial infrastructure. Although this framework may be useful for other clinical trial networks aiming to build resilient infrastructures, the generalizability of the lessons from the TOGETHER Trial described herein are limited by the geographic, social, and political context of the Minas Gerais state of Brazil. The emergence of COVID-19 as a global health threat and the urgent clinical response it demanded highlight the critical need for clinical trialists to build resilient trial infrastructures. Lessons learned from the TOGETHER Trial may help other trial networks respond quickly or more efficiently to future threats and may have implications for other trials or interventions.

Received November 17, 2021. Accepted for publication December 2, 2021.

Published online January 7, 2022.

Acknowledgments: We acknowledge our partners and funders in the preparation of this publication. The American Society of Tropical Medicine and Hygiene has waived the Open Access fee for this article due to the ongoing COVID-19 pandemic.

Financial support: Rainwater Charitable Foundation supported this research and has no role in the conduct of the research or findings of this publication.

Authors' addresses: Jamie I. Forrest, Platform Life Sciences, Vancouver, Canada, and University of British Columbia, Vancouver, 
Canada, E-mail: jforrest@platformsciences.ca. Angeli Rawat and Felipe Duailibe, University of British Columbia, Vancouver, Canada, E-mails: angeli@alumni.ubc.ca and felipetduailibe@hotmail.com. Christina M. Guo, Platform Life Sciences, Vancouver, Canada, and University of Aberdeen, Aberdeen, Scotland, E-mail: cguo@ platformsciences.ca. Sheila Sprague, McMaster University, Hamilton, Canada, E-mail: sprags@mcmaster.ca. Paula McKay, CardResearch, Belo Horizonte, Brazil, E-mail: mckayp@mcmaster. ca. Gilmar Reis, CardResearch, Belo Horizonte, Brazil, and Pontifica Católica Universidade de Minas Gerais, Belo Horizonte, Brazil, E-mail: greisbh@uol.com.br. Edward J. Mills, Platform Life Sciences, Vancouver, Canada, and McMaster University, Hamilton, Canada, E-mail: millsej@mcmaster.ca.

This is an open-access article distributed under the terms of the Creative Commons Attribution (CC-BY) License, which permits unrestricted use, distribution, and reproduction in any medium, provided the original author and source are credited.

\section{REFERENCES}

1. Thorlund K, Dron L, Park J, Hsu G, Forrest JI, Mills EJ, 2020. A real-time dashboard of clinical trials for COVID-19. Lancet Digit Health 2: e286-e287.

2. Pan $\mathrm{H}$ et al., 2021. Repurposed antiviral drugs for COVID-19: interim WHO Solidarity Trial results. N Engl J Med 384: 497511.

3. RECOVERY Collaborative Group, 2020. Lopinavir-ritonavir in patients admitted to hospital with COVID-19 (RECOVERY): a randomised, controlled, open-label, platform trial. Lancet 396: 1345-1352.

4. RECOVERY Collaborative Group, 2021. Tocilizumab in patients admitted to hospital with COVID-19 (RECOVERY): a randomised, controlled, open-label, platform trial. Lancet 397: 1637-1645.

5. RECOVERY Collaborative Group, 2021. Azithromycin in patients admitted to hospital with COVID-19 (RECOVERY): a randomised, controlled, open-label, platform trial. Lancet 397: 605-612.

6. Butler CC et al., 2021. Azithromycin for community treatment of suspected COVID-19 in people at increased risk of an adverse clinical course in the UK (PRINCIPLE): a randomised, controlled, open-label, adaptive platform trial. Lancet 397: 1063-1074.

7. Butler CC et al., 2021. Doxycycline for community treatment of suspected COVID-19 in people at high risk of adverse outcomes in the UK (PRINCIPLE): a randomised, controlled, openlabel, adaptive platform trial. Lancet Respir Med 9: 1010-1020.

8. Yu L-M et al., 2021. Inhaled budesonide for COVID-19 in people at high risk of complications in the community in the UK (PRINCIPLE): a randomised, controlled, open-label, adaptive platform trial. Lancet 398: 843-855.

9. Angus DC et al., 2020. Effect of hydrocortisone on mortality and organ support in patients with severe COVID-19. JAMA 324: 1317-1329.

10. REMAP-CAP, ACTIV-4a, and ATTACC Investigators, 2021. Therapeutic anticoagulation with heparin in critically ill patients with COVID-19. N Engl J Med 385: 777-789.

11. Reis $G$ et al., 2021. Effect of early treatment with hydroxychloroquine or lopinavir and ritonavir on risk of hospitalization among patients with COVID-19: the TOGETHER randomized clinical trial. JAMA Netw Open 4: e216468: 1010-1020.

12. Reis $\mathrm{G}$ et al., 2021. A multi-center, adaptive, randomized, platform trial to evaluate the effect of repurposed medicines in outpatients with early coronavirus disease 2019 (COVID-19) and high-risk for complications: the TOGETHER master trial protocol. Gates Open Res 5: 117.

13. Duley L et al., 2008. Specific barriers to the conduct of randomized trials. Clin Trials 5: 40-48.

14. Kruk ME, Myers M, Varpilah ST, Dahn BT, 2015. What is a resilient health system? Lessons from Ebola. Lancet 385: 19101912.

15. Lee Z, Rayner CR, Forrest JI, Nachega JB, Senchaudhuri E, Mills EJ, 2021. The rise and fall of hydroxychloroquine for the treatment and prevention of COVID-19. Am J Trop Med Hyg 104: 35-38.

16. Woodcock J, LaVange LM, 2017. Master protocols to study multiple therapies, multiple diseases, or both. $N$ Engl $J$ Med 377: 62-70.

17. Forrest JI, Rayner CR, Park JJH, Mills EJ, 2020. Early treatment of COVID-19 disease: a missed opportunity. Infect Dis Ther 9: 715-720. 